J. Lake Sci. (湖泊科学) , 2006, 18(6):590-596

http:// www. jlakes. org. E-mail: jlakes@ niglas. ac.cn

(c) 2006 by Journal of Lake Sciences

\title{
柴达木盆地苏干湖表层沉积与尘暴事件一一元素示踪的初步结果
}

\author{
强明瑞 ${ }^{1}$, 鲁瑞洁 ${ }^{2}, 弓{\text { 家武 }{ }^{1}, \text { 肖 舜 }{ }^{1}, \text { 陈发虎 }}^{1}$ \\ (1: 兰州大学西部环境教育部重点实验室 中德干旱环境联合研究中心,兰州 730000) \\ (2: 北京师范大学中国沙漠研究中心, 北京 100875)
}

\begin{abstract}
摘要: 通过分析苏干湖地区 10 个地表表层沉积物、9 个大气降尘(含 2 个尘暴降尘)、16 个湖泊表层沉积物等样品的 8 种常量 - 微 量元素和 11 种稀土元素的含量, 以及同类沉积不同元素含量和不同沉积同一元素含量的相关性, 结果表明, 在湖泊沉积体系中 $\mathrm{Al} 、 \mathrm{Fe} 、 \mathrm{Sc}$ 含量的变化与强风 - 尘暴过程有关, 指示了湖泊沉积碎屑颗粒风成输人的可能性. 元素 $\mathrm{Fe} 、 \mathrm{Sc}$ 含量在 3 种不同的沉积物 中差异不大, 与尘暴事件的强度和频次有关. 稀土元素结果显示 3 类沉积具有同源性. 苏干湖沉积可以用来重建研究区尘暴演化 历史.
\end{abstract}

关键词:尘暴事件;降尘;元素示踪;苏干湖

\section{Surface sediments of Lake Sugan and dust storm in the Northern Qaidam Basin, China : pre- liminary results of elemental tracers}

QIANG Mingrui ${ }^{1}$, LU Ruijie ${ }^{2}$, ZHANG Jiawu ${ }^{1}$, XIAO Shun ${ }^{1} \&$ CHEN Fahu ${ }^{1}$

(1: CAEP, Key Laboratory of Western Chinaś Environmental System, MOE, Lanzhou University, Lanzhou 730000, P. R. China)

(2: China Center of Desert Research at Beijing Normal University, Beijing Normal University, Beijing 100875, P. R. China)

Abstract: To understand the role of atmospheric dust in the global climate system further, it is necessary that a longer sequence of dust storm history should be established using geological archives in dust provenance area. Hydrologically closed lakes in the arid regions of China could be ideal objectives to trace dust storm evolution since the most of Northwest China has been recognized as an important source area for global dust loading. Lake Sugan, a closed lake located at the northern margin of the Qinghai-Tibetan Plateau, would be employed to study on the dust storm history in the Qaidam Basin. Here, 8 elements and 11 rare-earth elements were analyzed for three different sediments/deposits in the Sugan Lake region, namely, the surface sediments of Lake Sugan, the settled sand dust including materials collected during dust storm durations, and the surface deposits of the region. The results show the element of $\mathrm{Al}$ has been enriched by $11 \%$ relative to the surface ground deposits. The composition of the element in lake sediments is nearly half of the settled dust, which may be diluted by the carbonate accounting for about $50 \%$ of the lake sediment. Two of $\mathrm{Al}, \mathrm{Fe}$ and $\mathrm{Sc}$ exhibit apparent positive correlations in the lake sediments. Also, for one of the three elements positive correlation was found between the settled sand dust and the lake sediments. However, there are negative correlations between the catchment deposits and the settled sand dust, and between the catchment deposits and the lake sediment for Al and Sc. Variations in $\mathrm{Fe}$ and $\mathrm{Sc}$ compositions exhibit an apparent positive correlation not only with intensity of dust storm events, but also with frequency of the events. The Fe and Sc compositions seem to be changeless for the three kinds of sediments/ deposits. In addition, the results of rare-earth elements analyses show that the lake sediments, the settled sand dust and the surface ground deposits may derive from a homologous source. The elements of $\mathrm{Al}, \mathrm{Fe}, \mathrm{Sc}$ are most likely to be

* 国家自然科学基金项目(40301051, 40421101). 2005-11-30 收稿;2006-04-12 收修改稿. 强明瑞, 男, 1973 年生, 博士, 副教授; E-mail: mrqiang@lzu.edu.cn. 
transported into lake through strong wind and dust storm processes. Therefore, sediments of Lake Sugan could be used to trace dust storm in this region, and the elements of $\mathrm{Al}, \mathrm{Fe}$, Sc could be applied in reconstruction of dust storm history.

Keywords: Dust storm; the settled sand dust; elemental tracers; Lake Sugan

大气粉尘载荷与全球大气环流形势的改变密切相关, 因此地质载体记录的粉尘变化有助于揭示全球尺度的 气候事件 ${ }^{[1]}$. 我国西北干旱、半干旱地区被认为是全球大气粉尘的主要源区之一, 为格陵兰冰芯(雪)沉积、北太 平洋深海沉积等记录所证实 ${ }^{[1-3]}$. 尽管我国学者利用黄土沉积重建了较长历史的风尘通量或者大气粉尘载荷的 变化序列, 可是这些序列分辨率一般为千年尺度, 且记录分布在黄土高原 ${ }^{[4,5]}$. 目前, 有关我国干旱区 (粉尘源区) 高分辨率的粉尘序列尚无报道. 我国干旱区普遍遭受强烈的风蚀作用, 很难保存连续性良好的风成沉积. 湖泊沉 积物, 尤其是干旱 - 半干旱地区的封闭湖泊沉积, 可用来重建地质历史时期粉尘源区的尘暴演化或者风沙活动 历史 ${ }^{[6,7]}$.

近 20 年来, 元素示踪被有效地运用到大气粉尘源地和粉尘释放、输送与沉降过程的研究中 ${ }^{[8-10]}$. 张小曳等通 过粉尘元素浓度 - 粒度的组配特征成功示踪了我国粉尘的主要源区 ${ }^{[11,12]}$, 并利用黄土沉积中特征元素含量重建 了 $250 \mathrm{ka}$ 来亚洲大气粉尘载荷的变化历史 ${ }^{[4]}$. 然而, 利用干旱区封闭湖泊重建源区粉尘释放和尘暴演化历史的研 究还不多见, 而利用元素示踪手段判别湖泊沉积与粉尘释放或者尘暴过程的联系几乎没有. 鉴于此, 本文拟通过 分析柴达木盆地北部地表沉积物、大气降尘(尘暴降尘)、湖泊表层沉积物的元素组成, 初步确定了苏干湖沉积物 记录尘暴事件的元素组成联系,为进一步利用湖泊沉积物揭示尘暴演化历史提供依据.

\section{1 材料与方法}

苏干湖 $\left(38^{\circ} 51^{\prime} \mathrm{N}, 93^{\circ} 54^{\prime} \mathrm{E}\right)$ 盆地是嵌套在柴达木盆地北部的一个封闭盆地, 地势东高西低, 海拔高度变化在 $2800-3200 \mathrm{~m}$. 盆地西部年平均气温为 $2.75{ }^{\circ} \mathrm{C}$, 多年平均降水量为 $18.7 \mathrm{~mm}$, 蒸发量为 $2967.2 \mathrm{~mm}$, 属于极端干旱 区. 该地区大风天气盛行, $1957-2000$ 年年均大风日数为 $55 \mathrm{~d}$; 沙尘暴发生频率高, 同期年沙尘暴日数变化在 $3-$ $16 \mathrm{~d}$. 尘暴和大风日数多出现在 3-7 月, 盛行风向为 WNW. 盆地内降水量小,不足以产生较大的人湖径流. 盆地补 给水主要来自东部的大哈尔腾河和小哈尔腾河, 这两条河流在盆地东部山区为常年性河流. 河流到达山前平原戈 壁带之后, 在 8-15 km 的地段内即全部渗人地下, 在湖泊东部细土平原带以泉水出露,进而汇集成河流补给苏干 湖. 盆地植被稀少, 地下水出露的湖泊东部等地分布有草地, 其余均为戈壁、沙砾地和沙地等荒漠景观. 研究区人 类活动较少, 少许哈萨克人在该流域放牧. 苏干湖为封闭湖泊, 面积 $103.68 \mathrm{~km}^{2}$, 平均水深 $2.5 \mathrm{~m}$, 沉积中心水深约 $5.0 \mathrm{~m}$. 盆地南部马海湖钻孔资料表明自晚更新世以来苏干湖就封闭起来 ${ }^{[13]}$, 流域水文循环模式相对简单, 因此, 苏干湖是重建尘暴演化历史的理想地点.

2002 年 1 月,利用 Grab 抓斗获取 3-5 cm 深度的湖泊沉积物表层样品, 共 18 块 (包括小苏干湖样品 1 个, 该 湖位于苏干湖水系上游, 由地下水汇集而成). 流域内表层沉积物采集地点包括古湖岸堤、沙砾地、风成堆积、地表 径流冲积物和现代湖泊湖滩等,共 13 块样品. 2004 年 11 月,在苏干湖以西补充采集地表样品 37 块.

在距离苏干湖 $60 \mathrm{~km}$ 的冷湖气象站收集大气降尘样品, 包括尘暴期间的降尘. 在冷湖气象局房顶 (距地 面 $3.5 \mathrm{~m}$ ) 布置的 3 个降尘缸 $(\mathrm{D}=15 \mathrm{~cm}, \mathrm{H}=30 \mathrm{~cm}$ ), 底部铺设 2 层直径为 $1.5 \mathrm{~cm}$ 的玻璃球, 分别收集尘暴 事件降尘 (尘暴天气出现时, 打开降尘缸, 结束时关闭)、季节降尘和年降尘. 另一降尘缸 ( $\mathrm{D}=36 \mathrm{~cm}, \mathrm{H}=70$ $\mathrm{cm}$ ) 在同样高度收集月降尘. 每次搜集将降尘缸和玻璃球用蒸馏水冲洗干净, 然后将洗液在 $105^{\circ} \mathrm{C}$ 下烘干, 称重保存.

10 个湖泊表层样品、9 个降尘样品 (其中尘暴事件降尘 2 个、月降尘 2 个、季节降尘 4 个、年降尘 1 个) 以及 16 个流域表层样品用来做元素分析, 在北京师范大学分析测试中心完成. 将样品研磨, 过 100 目篮, 准 确称取 $0.1 \mathrm{~g}$, 置人聚四氟乙烯罐中, 加人 $3 \mathrm{ml}$ 硝酸、 $1 \mathrm{ml}$ 高氯酸、 $1 \mathrm{ml}$ 氢氟酸, 放于不锈钢外套中于烘箱中 $160^{\circ} \mathrm{C}$ 加热 $4-5 \mathrm{~h}$, 冷却后取出, 在电热板上敞口加热去硅及残留的氢氟酸, 待大量白烟冒尽, 样品呈可流动 球珠状时取下, 加人 $1 \mathrm{ml}$ 硝酸, 微热, 冷却至常温, 用高纯水定容至 $10 \mathrm{ml}$, 用法国生产 JY - ULTIMA 型电感 耦合等离子体原子发射光谱仪 (ICP - AES) 测定无机元素含量. 被测元素包括 W、Sr、Al、Fe、Mg、Ti、Zr、Sc, 稀 土元素 $(R E E): L a 、 C e 、 P r 、 N d 、 S m 、 E u 、 G d 、 D y 、 E r 、 Y b 、 Y$ 等. 元素含量以 $\mu \mathrm{g} / \mathrm{g}$ 表示, 分析精度 $<10 \%$. 


\section{2 结果与讨论}

\section{1 常量 - 微量元素}

湖泊表层沉积、降尘和流域地表沉积 8 种常量 - 微量元素含量的变化范围、平均值以及各元素含量的 变差系数 $(C v)$ 如表 1 、图 1 所示. 由于化学沉积盐的介人,湖泊沉积物富含 $\mathrm{Sr} 、 \mathrm{Mg}$ 等元素, 其平均含量明显 高于地表沉积和降尘 (表 1 ; 图 1a, c). 降尘中 8 种元素的 $C v$ 值变化在 $0.06-0.22$,除 $\mathrm{W}$ 外均小于湖泊表层 样品与流域地表样品, 表明大部分被测元素含量在降尘中的离散程度相对较小. 湖泊沉积与流域地表沉积 具有较大的 $C v$ 值, 可能与流域样品采集地点、机械组成和湖泊沉积含有较多的化学沉积有关. 湖泊沉积中 元素 $\mathrm{W} 、 \mathrm{Ti} 、 \mathrm{Al}$ 含量的变化范围小于降尘, 表现为降尘样品中含量最高, 平均值分别为 7.30、3011 和 241860 $\mu \mathrm{g} / \mathrm{g}$ (表 1), 分别较地表样品的平均值富集了 $30 \% 、 13 \% 、 11 \%$, 表明粉尘在释放 - 沉积过程中这些元素得 到了有效富集,可能与颗粒物的分选作用或者这些元素所赋存的矿物容易遭受风力吹扬有关. 元素 $\mathrm{Fe} 、 \mathrm{Sc}$ 在三类沉积物中变化范围相互重叠, $C v$ 值较大, 但平均值比较接近 (表 1 ). 元素 $\mathrm{Zr}$ 含量由流域地表沉积、 大气降尘至湖泊沉积物渐次变小 (表 1 ).

表 2 分析了湖泊表层沉积、降尘、流域地表沉积中 6 种元素 $(\mathrm{Al} 、 \mathrm{Ti} 、 \mathrm{~W} 、 \mathrm{Fe} 、 \mathrm{Sc} 、 \mathrm{Zr})$ 的相关性.湖泊表层沉 积中 6 种元素之间具有明显的正相关关系,均通过 $99 \%$ 的信度检验. 流域地表沉积中, Ti 与其他 5 种元素 具有显著正相关,通过 $95 \%$ 的信度检验; $\mathrm{Fe} 、 \mathrm{Sc} 、 \mathrm{Zr}$ 之间相关性显著,通过 $99 \%$ 的信度检验. 降尘样品中 Sc 与 $\mathrm{Al} 、 \mathrm{Ti} 、 \mathrm{Fe}$ 等元素显著正相关,通过 $95 \%$ 的信度检验,其他元素之间相关性较差. 湖泊沉积物中各元素的 关系表明至少这 6 种元素在湖泊沉积过程中行为相似. $\mathrm{Ti}$ 一般不形成可溶性的化合物 ${ }^{[14]}, \mathrm{Al}$ 被溶于水的量 也很少 ${ }^{[15]}$, 可见这些元素均可能陆源产生. 降尘中元素之间的相关性较差可能与粉尘释放和有效沉降过程 有关,期间粒度组成的变化也可能对元素产生一定的分馏效应,有待于进一步深人研究.

\section{表 1 湖泊表层沉积、降尘和地表沉积元素含量测试结果}

Tab. 1 Variations of the elemental compositions of the lake sediments, the settled sand dust and the surface deposits in the study area

\begin{tabular}{|c|c|c|c|c|c|c|c|c|c|}
\hline 样品 (个数) & 测量值 & $\mathrm{Al}^{(1)}$ & $\mathrm{Ti}^{(1)}$ & $\mathrm{W}^{(2)}$ & $\mathrm{Fe}^{(1)}$ & $\mathrm{Sc}^{(2)}$ & $\mathrm{Mg}^{(1)}$ & $\mathrm{Sr}^{(1)}$ & $\mathrm{Zr}^{(2)}$ \\
\hline \multirow[t]{3}{*}{ 湖泊表层沉积 $(10)$} & )变化范围 & $94.75-190.60$ & $1.25-2.53$ & $2.57-3.61$ & $15.92-32.89$ & $11.76-22.78$ & $44.87-71.65$ & $2.24-3.33$ & $59.55-142.40$ \\
\hline & 平均值 & 133.00 & 1.79 & 3.40 & 23.43 & 16.70 & 57.64 & 2.84 & 85.70 \\
\hline & $C v^{(3)}$ & 0.26 & 0.28 & 0.22 & 0.24 & 0.24 & 0.15 & 0.14 & 0.30 \\
\hline \multirow[t]{3}{*}{ 降尘 (9) } & 变化范围 & $203.70-255.50$ & $2.78-3.27$ & $5.78-10.21$ & $19.77-25.83$ & $15.56-20.09$ & $26.56-38.98$ & $0.72-0.83$ & $85.48-118.1$ \\
\hline & 平均值 & 241.86 & 3.01 & 7.30 & 23.16 & 18.00 & 32.70 & 0.76 & 103.10 \\
\hline & $C v$ & 0.06 & 0.05 & 0.22 & 0.10 & 0.08 & 0.12 & 0.06 & 0.12 \\
\hline \multirow[t]{3}{*}{ 地表沉积 (16) } & 变化范围 & $125.70-299.30$ & $1.61-4.09$ & $4.15-7.10$ & $13.93-40.23$ & $10.97-31.48$ & $14.56-93.69$ & $0.51-1.95$ & $61.45-297.70$ \\
\hline & 平均值 & 217.56 & 2.67 & 5.60 & 24.59 & 19.30 & 38.80 & 1.07 & 113.00 \\
\hline & $C v$ & 0.19 & 0.28 & 0.17 & 0.38 & 0.37 & 1.03 & 0.37 & 0.53 \\
\hline
\end{tabular}

(1)元素含量单位为 $\times 10^{3} \mu \mathrm{g} / \mathrm{g}$; (2)单位为 $\mu \mathrm{g} / \mathrm{g}$; (3)为变差系数.

不同沉积中同一元素的相关性分析表明 (表 2)，Al、Ti、W、Fe、Sc、Zr 等元素含量在地表沉积与湖泊沉 积之间具有负相关关系,相关系数变化在 -0.24 至 -0.60 之间. 降尘与湖泊沉积中元素 $\mathrm{Al}$ 表现为良好的 正相关关系,达到 $95 \%$ 的信度水平; $\mathrm{Fe} 、 \mathrm{Sc} 、 \mathrm{Zr}$ 也表现为正相关关系. 降尘与地表沉积中 $\mathrm{Al} 、 \mathrm{Ti} 、 \mathrm{Sc}$ 含量呈负 相关关系. 考虑极端情况,如果湖泊碎屑沉积以河流输人为主,那么地表沉积与湖泊表层沉积的元素含量应 该是正相关关系,尽管元素含量受到湖泊内生沉积物的稀释作用. 因此,就不同沉积中元素的自相关性而 言, 湖泊沉积中风成组分的输人不得不考虑. 元素 $\mathrm{Al}$ 在地表沉积和降尘中呈负相关, 在降尘与湖泊沉积中 呈正相关关系. 前面的分析表明, $\mathrm{Al}$ 由地表沉积到降尘物质中平均含量富集了 $11 \%$,支持了地表沉积与降 尘之间 $\mathrm{Al}$ 的负相关关系. 湖泊沉积中 $\mathrm{Al}$ 的平均含量为 $133000 \mu \mathrm{g} / \mathrm{g}$ (表 1), 为降尘中含量的 $54 \%$, 比降尘 样品低了近一半. 用于元素测试的湖泊样品是全样, 表层沉积物碳酸盐含量约为 $54 \%$, 考虑到测试误差和 湖泊沉积物中较降尘物质富含有质机等因素, $\mathrm{Al}$ 含量近一半的减少应该是碳酸盐等沉积的稀释作用所致. 
表 2 湖泊表层沉积、降尘物质、流域表层沉积等同类样品中各元素含量的相关性分析 以及不同类型沉积同一元素的相关性分析 ${ }^{1)}$

Tab. 2 Correlation analyses on element compositions of the surface lake sediments, the settled sand dust and the surface deposits in catchment, respectively, and correlation analyses on a same element among the different sediments/deposits

\begin{tabular}{|c|c|c|c|c|c|c|c|c|c|c|c|c|c|c|c|c|c|c|c|}
\hline & & & $\mathrm{Al}$ & & & $\mathrm{Ti}$ & & & W & & & $\mathrm{Fe}$ & & & $\mathrm{Sc}$ & & & $\mathrm{Zr}$ & \\
\hline & & $\mathrm{L}$ & $\mathrm{D}$ & CS & L & $\mathrm{D}$ & CS & L & $\mathrm{D}$ & CS & $\mathrm{L}$ & $\mathrm{D}$ & CS & $\mathrm{L}$ & D & CS & $\mathrm{L}$ & $\mathrm{D}$ & CS \\
\hline \multirow[t]{4}{*}{$\mathrm{AI}$} & $\mathrm{L}$ & 1 & & & & & & & & & & & & & & & & & \\
\hline & $\mathrm{D}$ & $\begin{array}{c}0.66 \\
/ 0.05\end{array}$ & 1 & & & & & & & & & & & & & & & & \\
\hline & CS & $\begin{array}{l}-0.45 \\
/ 0.22\end{array}$ & $\begin{array}{l}-0.58 \\
/ 0.10\end{array}$ & 1 & & & & & & & & & & & & & & & \\
\hline & $\mathrm{L}$ & $\begin{array}{l}0.99 \\
/ 0.01\end{array}$ & & & 1 & & & & & & & & & & & & & & \\
\hline \multirow[t]{3}{*}{$\mathrm{Ti}$} & $\mathrm{D}$ & & $\begin{array}{c}0.19 \\
/ 0.63\end{array}$ & & 0 & 1 & & & & & & & & & & & & & \\
\hline & CS & & & $\begin{array}{c}0.66 \\
/ 0.05\end{array}$ & $\begin{array}{l}-0.58 \\
/ 0.11\end{array}$ & $\begin{array}{l}-0.17 \\
/ 0.66\end{array}$ & 1 & & & & & & & & & & & & \\
\hline & $\mathrm{L}$ & $\begin{array}{c}0.93 \\
/ 0.01\end{array}$ & & & $\begin{array}{c}0.89 \\
/ 0.01\end{array}$ & & & 1 & & & & & & & & & & & \\
\hline \multirow[t]{3}{*}{ W } & $\mathrm{D}$ & & $\begin{array}{l}-0.57 \\
/ 0.11\end{array}$ & & & $\begin{array}{l}-0.60 \\
/ 0.09\end{array}$ & & $\begin{array}{l}-0.53 \\
/ 0.14\end{array}$ & 1 & & & & & & & & & & \\
\hline & CS & & & $\begin{array}{c}0.94 \\
/ 0.01\end{array}$ & & & $\begin{array}{l}0.67 \\
/ 0.05\end{array}$ & $\begin{array}{l}-0.24 \\
/ 0.54\end{array}$ & $\begin{array}{l}0.26 \\
0.50\end{array}$ & 1 & & & & & & & & & \\
\hline & $\mathrm{L}$ & $\begin{array}{c}0.96 \\
/ 0.01\end{array}$ & & & $\begin{array}{c}0.95 \\
/ 0.01\end{array}$ & & , & $\begin{array}{c}0.96 \\
/ 0.01\end{array}$ & & & 1 & & & & & & & & \\
\hline \multirow[t]{3}{*}{$\mathrm{Fe}$} & $\mathrm{D}$ & & $\begin{array}{l}0.48 \\
/ 0.20\end{array}$ & & & $\begin{array}{l}0.48 \\
/ 0.20\end{array}$ & & & $\begin{array}{l}-0.31 \\
0.42\end{array}$ & & $\begin{array}{c}0.36 \\
/ 0.34\end{array}$ & 1 & & & & & & & \\
\hline & CS & & & $\begin{array}{l}0.46 \\
/ 0.21\end{array}$ & & & $\begin{array}{c}0.92 \\
/ 0.01\end{array}$ & & & $\begin{array}{l}0.46 \\
/ 0.22\end{array}$ & $\begin{array}{l}-0.51 \\
/ 0.17\end{array}$ & $\begin{array}{l}0.33 \\
/ 0.33\end{array}$ & 1 & & & & & & \\
\hline & $\mathrm{L}$ & $\begin{array}{c}0.99 \\
/ 0.01\end{array}$ & & & $\begin{array}{c}0.99 \\
/ 0.01\end{array}$ & & & $\begin{array}{c}0.94 \\
/ 0.01\end{array}$ & & & $\begin{array}{l}0.99 \\
/ 0.01\end{array}$ & & & 1 & & & & & \\
\hline \multirow[t]{3}{*}{$\mathrm{Sc}$} & $\mathrm{D}$ & & $\begin{array}{l}0.76 \\
/ 0.02\end{array}$ & & & $\begin{array}{c}0.72 \\
/ 0.03\end{array}$ & & & $\begin{array}{l}-0.68 \\
0.04\end{array}$ & & & $\begin{array}{l}0.75 \\
/ 0.02\end{array}$ & & $\begin{array}{c}0.49 \\
/ 0.18\end{array}$ & 1 & & & & \\
\hline & CS & & & $\begin{array}{c}0.50 \\
/ 0.18\end{array}$ & & & $\begin{array}{c}0.93 \\
/ 0.01\end{array}$ & & & $\begin{array}{c}0.43 \\
/ 0.24\end{array}$ & & & $\begin{array}{l}0.95 \\
/ 0.01\end{array}$ & $\begin{array}{l}-0.60 \\
/ 0.09\end{array}$ & $\begin{array}{l}-0.35 \\
/ 0.35\end{array}$ & 1 & & & \\
\hline & $\mathrm{L}$ & $\begin{array}{c}0.94 \\
/ 0.01\end{array}$ & & & $\begin{array}{c}0.89 \\
/ 0.01\end{array}$ & & & $\begin{array}{l}0.91 \\
/ 0.01\end{array}$ & & & $\begin{array}{c}0.89 \\
/ 0.01\end{array}$ & & & $\begin{array}{c}0.91 \\
/ 0.01\end{array}$ & & & 1 & & \\
\hline \multirow[t]{2}{*}{$\mathrm{Zr}$} & $\mathrm{D}$ & & $\begin{array}{l}-0.19 \\
/ 0.62\end{array}$ & & & $\begin{array}{l}0.36 \\
/ 0.35\end{array}$ & & & $\begin{array}{c}0.49 \\
/ 0.18\end{array}$ & & & $\begin{array}{c}0.25 \\
/ 0.52\end{array}$ & & & $\begin{array}{c}0.01 \\
/ 0.80\end{array}$ & & $\begin{array}{l}0.25 \\
/ 0.52\end{array}$ & 1 & \\
\hline & $\mathrm{CS}$ & & & $\begin{array}{c}0.57 \\
/ 0.12 \\
\end{array}$ & & & $\begin{array}{c}0.98 \\
/ 0.01 \\
\end{array}$ & & & $\begin{array}{c}0.60 \\
/ 0.09 \\
\end{array}$ & & & $\begin{array}{c}0.95 \\
/ 0.01 \\
\end{array}$ & & & $\begin{array}{c}0.93 \\
/ 0.01 \\
\end{array}$ & $\begin{array}{l}-0.56 \\
/ 0.12 \\
\end{array}$ & $\begin{array}{c}0.03 \\
/ 0.93 \\
\end{array}$ & 1 \\
\hline
\end{tabular}

1) 表中 L、D、CS 分别代表湖泊表层沉积、降尘、流域地表沉积, “/”后数字为置信水平. 相关分析样本数均 为 9 .

事实上, 苏干湖流域现代水文过程相对简单, 人湖径流主要是由地下水出渗汇集而成的次生河流,经湖泊东面的 沼泽地带人湖,其夹沙能力有限. 根据湖泊表层沉积物粒度组成的空间分布特征,至少较粗颗粒的碎屑组分由河 流输人的机会很小 ${ }^{1}$. 流域年降水量稀少, 不足以产生暂时性径流携带碎屑颗粒人湖. 2 次典型尘暴事件降尘中 $\mathrm{Al}$ 含量随尘暴事件的强度有明显的富集 (表 3). 如果将苏干湖理解为一个巨大的“降尘收集器”, 那么强风或者尘暴 天气出现时, 必然接纳降尘物质. 另一方面, 用于元素测试的湖泊表层样品基本覆盖湖泊, 即便河流输入组分影响

(1) Qiang M R, Chen F H, Zhang J W et al. , 2006. Grain size in sediments from Lake Sugan: a possible linkage to dust storm events at the northern margin of the Qinghai-Tibetan Plateau. Environmental Geology (in press). 
到湖泊沉积元素含量的变化, 也不至于影响整个湖泊, 河流携带的碎屑物质由于水动力下降, 大量沉降在湖泊边 缘地带. 地表沉积与湖泊沉积之间元素含量所表现的负相关性,应该是由于粉尘自地表释放时所产生的分异,譬 如 $\mathrm{Al}$ 、Sc. 由此可见,元素 $\mathrm{Al}$ 在苏干湖沉积体系中的变化表明,至少该元素的输人主要由风力搬运,可能意味着湖 泊沉积碎屑颗粒由强风 - 尘暴过程输人为主.

湖泊沉积物中元素 $\mathrm{Fe}$ 和 $\mathrm{Sc}$ 的含量与降尘和地表沉积基本一致: $\mathrm{Fe}$ 含量分别为 $23431 、 23161 、 24588 \mu \mathrm{g} / \mathrm{g} ; \mathrm{Sc}$ 为 $16.7 、 18.0$ 和 $19.3 \mu \mathrm{g} / \mathrm{g}$ (表 1, 图 1), 并没有表现出类似于元素 $\mathrm{Al}$ 被湖泊碳酸盐沉积稀释近一半的事实. 这可 能与铁在溶液中的化学沉积作用有关: 铁在湖泊中由于 $\mathrm{Ph}$ 或者 $\mathrm{Eh}$ 值的改变, 从而以氧化物、氢氧化物、硅酸盐、 碳酸盐、磷酸盐、硫化物及硫酸盐等化合物的形式进入沉积物 ${ }^{[16]}$. 这两种元素含量的变化显著响应于尘暴事件的 强度和频次. 比较发生于 2003 年 11 月 30 日和 2004 年 9 月 11 日的 2 次尘暴事件(表 3 ), 事件 2 持续时间为 34 $\min , 10-\min$ 风速平均值为 $16.7 \mathrm{~m} / \mathrm{s}$, 降尘量为 $4.25 \mathrm{~g}$, 事件 2 的强度超过了事件 1 . 事件 2 降尘的 $\mathrm{Fe} 、 \mathrm{Sc}$ 含量分别 为 25830 和 $19.61 \mu \mathrm{g} / \mathrm{g}$, 较事件 1 富集了 $12.6 \%$ 和 $14.3 \%$, 表明这两种元素随尘暴强度的增加而明显富集. 元素 $\mathrm{Fe} 、 \mathrm{Sc}$ 的含量的变化与尘暴发生的频次也存在明显的正相关关系,相关系数分别为 0.92 和 $0.96(n=4)$, 而其他元 素和尘暴发生的频次没有显著关系 (表 4). 因此,元素 Fe 和 Sc 不但可以示踪尘暴事件的强度,而且可以示踪尘暴 事件发生频次. 由前面分析可知, Al、Fe、Sc 各自在降尘和湖泊沉积之间不但具有正相关关系,而且它们在湖泊沉 积中也表现出显著正相关. 事实上,中国沙漠大气中 $\mathrm{Al} 、 \mathrm{Fe} 、 \mathrm{Sc} 、 \mathrm{Ti}$ 等被证明是源于沙漠粉尘,是可选择的示踪元 素 ${ }^{[11]}$. 因此,元素 $\mathrm{Al} 、 \mathrm{Fe} 、 \mathrm{Sc}$ 含量在苏干湖沉积体系中的变化应该能够指示区域尘暴事件的发生情况.

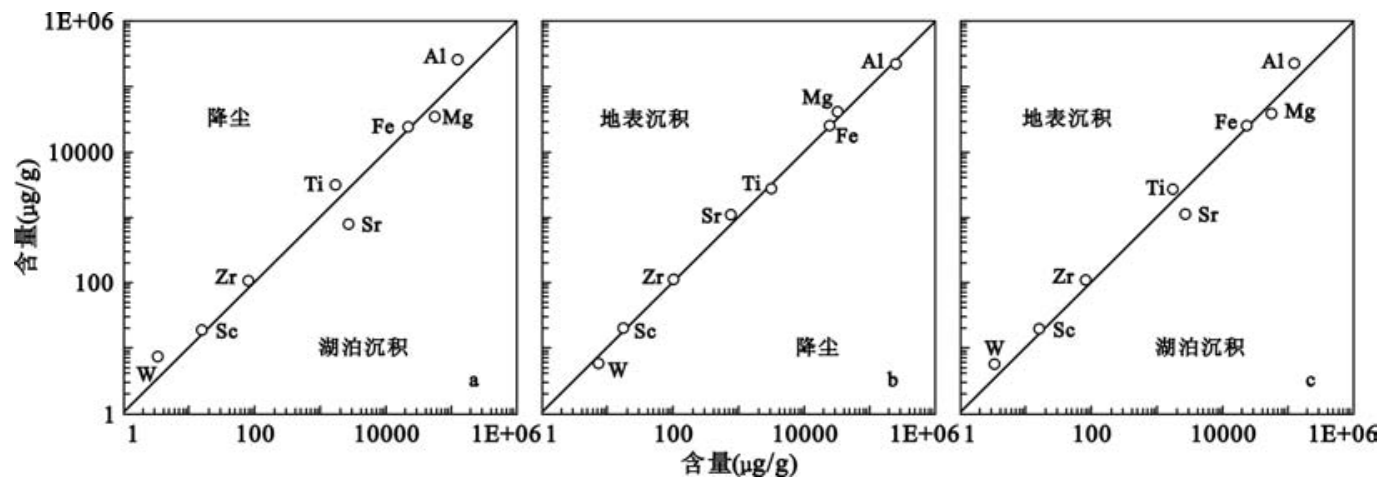

图 1 湖泊表层沉积、大气降尘和流域地表沉积物元素的比较

Fig. 1 Comparison of the major and trace element compositions among the surfacelake sediments, the settled dust and the surface deposits in the study area

表 32 次尘暴事件及其降尘元素 $\mathrm{Fe} 、 \mathrm{Sc} 、 \mathrm{Al} 、 \mathrm{Zr}$ 含量的比较

Tab. 3 Comparison of the two dust storms and the Fe, Sc, $\mathrm{Al}$ and $\mathrm{Zr}$ compositions of the settled sand dust collected within the two events

\begin{tabular}{|c|c|c|c|c|c|c|c|c|c|}
\hline 事件 & 日期 & $\begin{array}{c}\text { 持续时间 } \\
(\min )\end{array}$ & $\begin{array}{c}10-\min \text { 风速 } \\
\text { 平均值 }(\mathrm{m} / \mathrm{s})\end{array}$ & $\begin{array}{c}\text { 降尘量 } \\
(\mathrm{g})\end{array}$ & $\begin{array}{c}\text { 能见度 } \\
(\mathrm{m})\end{array}$ & $\begin{array}{c}\mathrm{Fe} \\
(\mu \mathrm{g} / \mathrm{g})\end{array}$ & $\begin{array}{c}\text { Sc } \\
(\mu \mathrm{g} / \mathrm{g})\end{array}$ & $\begin{array}{c}\mathrm{Al} \\
(\mu \mathrm{g} / \mathrm{g})\end{array}$ & $\begin{array}{c}\mathrm{Zr} \\
(\mu \mathrm{g} / \mathrm{g})\end{array}$ \\
\hline 12 & $2003-11-30$ & 107 & 16.1 & 2.78 & - & 22940 & 17.16 & 243900 & 85.48 \\
\hline 22 & $2004-09-11$ & 34 & 16.7 & 4.25 & 100 & 25830 & 19.61 & 255500 & 111.2 \\
\hline
\end{tabular}

\section{2 稀土元素}

11 种稀土元素 (rare-earth elements) 分析结果显示 (表 5), 流域地表稀土元素的绝对含量较降尘和湖泊沉积 物偏高, 降尘稀土含量又较湖泊沉积偏高. 稀土元素的 LREE/HREE 比值可能受到风化、沉积和成岩作用的影响, 但主要是反映其来源物质的特征 ${ }^{[17]}$. 湖泊沉积和降尘的 LREE/HREE 比值非常接近, 平均值分别为 2.572 和 2.564. 研究区地表样品的 LREE/HREE 比值为 2.699 , 意味着流域地表相对富集轻稀土. 地表样品的采集时, 轻轻 
刮去表层, 样品采集深度约 1-2 cm, 地表沉积尚未经过充分的风蚀作用. 苏干湖地区为强风蚀地区, 2 个季节降尘 样品的粒度分析显示, $100-200 \mu \mathrm{m}$ 颗粒约占 40\%, 10-20 $\mu \mathrm{m}$ 约占 3.7 -5.8\%, 中值粒径为 $131.3 \mu \mathrm{m}$. 研究区地 表沉积相对降尘样品粘土含量较大, 因而富集了轻稀土 ${ }^{[18]}$. 降尘样品较低的 LREE/HREE 表明大量的细粒物质 在风力吹扬下到达更遥远的地点沉降. 湖泊沉积轻、重稀土含量均低于降尘, 而 LREE/HREE 比值的一致性表明 湖泊沉积物与大气降尘具有同源性. 湖泊沉积物中稀土绝对含量低于其他两类样品, 可能与湖泊沉积物中大量化 学沉积的稀释作用有关.

表 4 不同季节尘暴频次与元素含量的关系

Tab. 4 Relationship between the frequency of dust storm events and the elemental compositions during different seasons

\begin{tabular}{lcccccccc}
\hline 月.日一月.日 & 频次 $(\mathrm{d})$ & $\mathrm{Fe}(\mu \mathrm{g} / \mathrm{g})$ & $\mathrm{Sc}(\mu \mathrm{g} / \mathrm{g})$ & $\mathrm{Al}(\mu \mathrm{g} / \mathrm{g})$ & $\mathrm{W}(\mu \mathrm{g} / \mathrm{g})$ & $\mathrm{Mg}(\mu \mathrm{g} / \mathrm{g})$ & $\mathrm{Sr}(\mu \mathrm{g} / \mathrm{g})$ & $\mathrm{Ti}(\mu \mathrm{g} / \mathrm{g})$ \\
\hline $11.3-1.4$ & 1 & 21530 & 17.59 & 241000 & 7.36 & 33100 & 834.2 & 3052 \\
$2.4-4.4$ & 4 & 24840 & 17.69 & 240000 & 8.77 & 38980 & 808.3 & 2936 \\
$5.4-7.4$ & 9 & 25760 & 19.81 & 249000 & 5.90 & 33540 & 770.9 & 3116 \\
$8.4-10.4$ & 1 & 21440 & 17.03 & 249700 & 5.95 & 27580 & 752.0 & 2816 \\
\hline
\end{tabular}

表 5 不同沉积物稀土元素含量平均值 (单位: $\mu \mathrm{g} / \mathrm{g}$ )

Tab. 5 Mean values of rare-earth elemental compositions of the different sediments ( Unit: $\mu \mathrm{g} / \mathrm{g}$ )

\begin{tabular}{ccccccccccccc}
\hline 沉积物 & $\mathrm{La}$ & $\mathrm{Ce}$ & $\mathrm{Pr}$ & $\mathrm{Nd}$ & $\mathrm{Sm}$ & $\mathrm{Eu}$ & $\mathrm{Gd}$ & $\mathrm{Dy}$ & $\mathrm{Er}$ & $\mathrm{Yb}$ & $\mathrm{Y}$ & LREE/HREE \\
\hline 湖泊沉积 & 15.71 & 31.92 & 4.76 & 21.36 & 5.25 & 0.85 & 5.78 & 2.27 & 1.28 & 1.14 & 20.57 & 2.572 \\
降尘 & 21.06 & 36.96 & 5.01 & 23.24 & 6.42 & 1.09 & 7.29 & 3.18 & 1.29 & 1.55 & 23.26 & 2.564 \\
地表沉积 & 21.18 & 41.01 & 6.25 & 23.19 & 7.27 & 1.15 & 7.63 & 3.17 & 1.23 & 1.48 & 23.56 & 2.699 \\
\hline
\end{tabular}

将稀土含量的平均值标准化 (与球粒陨石比 较) 得到 3 类样品稀土含量的配分曲线 (图 2), 湖泊 沉积稀土配分曲线除元素 $\mathrm{Er}$ 外,基本平行并且位于 降尘和地表沉积样品曲线的下方. 可见这 3 种沉积 的稀土分布模式基本一致, 反映了湖泊沉积物的物 源与研究区地表以及尘暴过程存在密切的联系, 支 持了常量 - 微量元素的分析结果, 表明利用苏干湖 沉积物重建该区的尘暴演化历史是可行的.

\section{3 结语}

苏干湖地处我国极端干旱区, 流域水文循环过 程相对简单. 通过分析流域地表沉积、大气降尘与湖 泊表层沉积元素含量的变化以建立湖泊沉积与强 风-尘暴过程的联系. 本文稀土元素的分析结果表 明,湖泊表层沉积与降尘和地表沉积具有同源性. 元 素 $\mathrm{Al}$ 在降尘中明显富集, 而湖泊沉积 $\mathrm{Al}$ 含量约为 降尘的一半,可能为湖泊表层沉积的碳酸盐稀释所

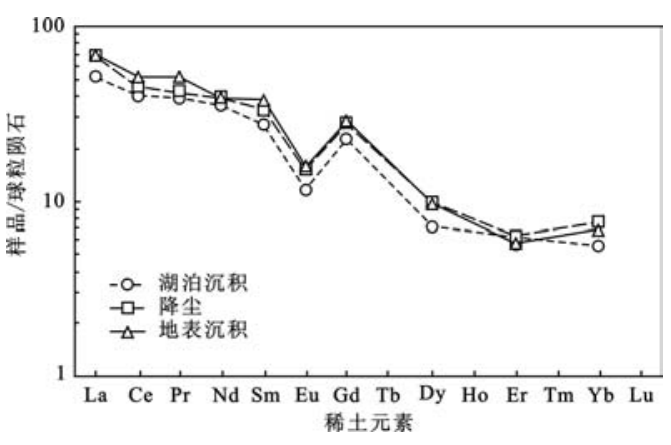

图 2 湖泊表层样品、降尘样品以及地表样品 稀土元素配分曲线 (与球粒陨石比较)

Fig. 2 Comparison of rare-earth elemental (REE) distribution patterns among the surface lake sediments, the settled sand dust and the surface deposits in the study area 致. $\mathrm{Al}$ 含量在降尘与地表沉积中表现为负相关,而在降尘与湖泊沉积中表现为正相关. Fe、Sc 分别在降尘与 湖泊沉积之间存在正相关性, 而且 Sc 在降尘与地表沉积之间存在负相关关系. 元素 Fe、Sc 明显指示了尘暴 事件的强度和频次. 然而, 这两个元素在湖泊沉积物中没有受到明显的稀释作用,可能与它们在湖泊中的地 球化学行为有关, 仍需要进一步开展工作. $\mathrm{Al} 、 \mathrm{Fe} 、 \mathrm{Sc}$ 等元素在不同沉积中含量的变化以及它们与尘暴事件 
强度或者频次的关系表明,湖泊沉积中碎屑组分风成输人显著,而且与尘暴过程联系密切. 可见, 苏干湖可 以用来重建区域尘暴演化历史, 元素 $\mathrm{Al} 、 \mathrm{Fe} 、 \mathrm{Sc}$ 可能被运用到湖泊岩芯中作为指示尘暴演化的替代指标. 拟将利用湖泊沉积物的碎屑组分 (扣除化学沉积与有机质组分) 开展元素测试,并结合碎屑矿物组成和风 成颗粒表面形态特征, 以判别湖泊沉积碎屑物质与降尘物质之间的元素组成联系及其对尘暴事件的响应. 致谢: 青海省冷湖行政委员会气象局协助收集降尘样品; 北京师范大学分析测试中心分析元素含量, 在此 致以谢忱.

\section{4 参考文献}

[1] Biscaye P E, Grousset P E, Revel M, et al. Asian provenance of glacial dust (stage 2) in the GISP2 ice core, Summit, Greenland. Journal of Geophysical Research, 1997, 102 ( C12) : 26675 -26781.

[2] Bory A J-M, Biscaye P E, Svensson A, et al. Seasonal variability in the origin of recent atmospheric mineral dust at NorthGRIP, Greenland. Earth and Planetary Science Letters, 2002, 196 : 123 -134.

[3] Pettke T, Halliday A N, Hall C M, et al. Dust production and deposition in Asia and the north Pacific Ocean over the past 12 Myr. Earth and Planetary Letters, 2000, 178: 397 -413.

[4] Zhang X Y, Lu H Y, Arimoto R, et al. Atmospheric dust loading and their relationship to rapid oscillations of the Asian winter monsoon climate: two 250-kyr loess records. Earth and Planetary Letters, 2002, 202 : $637-643$.

[5] 安芷生, 肖举乐. 黄土高原风尘通量研究的一个实例. 科学通报, 1990, 35(3): $220-223$.

[6] Street-Perrott F A, Holmes J A, Waller M P, et al. Drought and dust deposition in the West African Sahel: a 5500-year record from Kajemarum Oasis, northeastern Nigeria. The Holocene, 2000, 10 (3) : 293 -302.

[7] 金章东, 王苏民, 沈 吉等. 岱海地区近 400 年来的 “尘暴”事件一一来自岱海沉积物粒度的证据. 湖泊科学, 2000, 12 (3) : $193-197$.

[8] Duce R A, Arimoto R, Ray B J, et al. Atmospheric trace elements at Enewetak Atoll, 1. Concentrations, sources and temporal variability. Journal of Geophysical Research, 1983, 88: 5321 -5342.

[9] Parrington J R, Zoller W H, Aras N K. Asian dust: Seasonal Transport to the Hawaiian Island. Science, 1983, 220:195 -197.

[10] Arimoto R, Duce R A, Ray B J, et al. Trace elements in the atmosphere over the North Atlantic. Journal of Geophysical Research, 1995, 100: 1199 -1213.

[11] 张小曳, 张光宇, 朱光华等. 中国粉尘源区的元素示踪. 中国科学 (D 辑), 1996, 26 (5): $423-430$.

[12] 张小曳, 沈志宝, 张光宇等. 青藏高原远源西风粉尘与黄土堆积. 中国科学 (D 辑), 1996, 26 (2) : $147-153$.

[13] 刘淑琴, 张发胜. 柴达木马海盆地沉积环境和成盐作用. 北京: 地质出版社, 1992: 9 -12.

[14] 刘英俊, 曹励明, 元素地球化学导轮. 北京: 地质出版社, 1987: 128 .

[15] 牟保磊. 元素地球化学. 北京: 北京大学出版社, 1999: $95-96$.

[16] 中国科学院地球化学研究所. 铁的地球化学. 北京: 科学出版社, 1981: 85 -94.

[17] 文启忠等. 中国黄土地球化学. 北京: 科学出版社, 1989: 105 .

[18] 张虎才. 武都黄土剖面稀土元素研究. 地球化学, 1996, 25 (6) : 545 -551. 